\title{
ESPECIFICACIÓN DE UN MODELO DE HIPERMETROPÍA SOCIOPOLÍTICA
}

\author{
Cruz García Lirios ${ }^{\underline{1}}$ \\ Javier Carreón Guillén ${ }^{2}$ \\ Jorge Hernández Valdés ${ }^{3}$ \\ José Marcos Bustos Aguayo ${ }^{4}$ \\ José Alfonso Aguilar Fuentes ${ }^{5}$
}

Recibido el 11 de noviembre de 2014, aprobado el 16 de junio de 2015 y actualizado el 10 de noviembre de 2015

DOI: 10.17151/luaz.2016.42.17

\section{RESUMEN}

El proceso que consiste en asegurar que los desastres naturales y catástrofes ecológicas lejanas no están vinculados con la situación personal, o bien, las problemáticas locales no son de tal magnitud que supongan una acción conservacionista, es conocido como hipermetropía ambiental. En este sentido, el presente trabajo se propone especificar un modelo para el estudio de este sesgo perceptual que explica la relación entre naturaleza y humanidad. Para tales fines, se llevó a cabo un estudio documental en las bases de datos de RADALYC, LATINDEX y DIALNET durante el periodo que va de 2010 a 2014 en artículos con registro ISSN y DOI relativos a la hipermetropía ambiental. El modelo especificado incluye ocho dimensiones alusivas al sesgo perceptual con respecto al abastecimiento de agua local y residencial. En referencia al estado del conocimiento, la especificación fue contrastada a fin de poder anticipar escenarios de análisis, estructuras perceptuales, toma de decisiones y comportamientos en función de la disponibilidad de agua y las necesidades/expectativas de consumo locales.

\section{PALABRAS CLAVE}

Catástrofes ambientales, desastres naturales, abastecimiento de agua, percepción de riesgos, comportamientos proambientales.

\section{SPECIFICATION OF A SOCIOPOLITICAL HYPEROPIA MODEL}

\section{ABSTRACT}

The process consisting in ensuring that natural disasters and distant environmental catastrophes are not linked to the personal situation, or local issues are not of such magnitude thay they involve a conservation action, is known as environmental hyperopia. In this sense, the present article aims to specify a model for the study of this perceptual bias that explains the relationship between nature and humanity. To that end, a documental study was carried on RADALYC, DIALNET and LATINDEX databases during the period 2010 to 2014 in Articles with ISSN and DOI record concerning environmental hyperopia. The model specified includes eight dimensions referring the perceptual bias with respect to local and residential water supply. In reference to the state of knowledge, the specification was proven to be able to anticipate analysis scenarios, perceptual structures, decision-making and behavior in terms of water availability and the needs / expectations of local consumption. 
KEY WORDS: environmental catastrophes, natural disasters, water supply, perception of risk,. Pro-environmental behaviors

\section{INTRODUCCIÓN}

En Psicología de la Sustentabilidad, la hipermetropía, en su concepción general, supone percepciones sesgadas relativas al impacto de catástrofes ambientales y desastres naturales derivados del cambio climático que son apreciados por la sociedad como eventos aislados que no repercutirían ni directa, ni indirectamente en la calidad de vida, el confort residencial y el bienestar subjetivo (véase Tabla 1 en Anexo).

Corral et al. (2008), en el contexto de la psicología de los recursos y servicios hídricos, encontraron que las creencias utilitarias determinan el consumo de agua indicado por el lavado de trastos, aseo personal, riego de plantas, lavados de trastes y limpieza de acera. Es decir, la información concerniente a las sequías, escasez y desabastecimiento parece no incidir sobre las creencias que procesan la información de un modo tal que el agua es considerada un instrumento de limpieza, confort y esparcimiento. La hipermetropía parece ser un proceso complejo que estaría indicado por su grado de utilitarismo.

De tal manera, la hipermetropía antecede a las creencias utilitarias, pero en relación con los sistemas de procesamiento y categorización de información suponen, para Corral et al. (2006), otras relaciones con percepciones relativas a la perspectiva del tiempo: 1) orientación al pasado, 2) orientación al futuro y 3) estilos sustentables con dimensiones tales como: a) presente hedonista, b) presente fatalista, c) pasado positivo, d) pasado negativo y e) propensión al futuro. Cada una de las dimensiones, al interactuar entre ellas con la finalidad de anticipar la conservación de agua, estableció un sistema perceptual y conductual en el que la hipermetropía estaría vinculada con orientación al pasado y las dimensiones del presente hedonista y el pasado positivo. Es decir, la hipermetropía sería un proceso de información concerniente a riesgos ubicados en el pasado, que estarían poco relacionados con el presente de confort y desvinculados del futuro.

No obstante, si la hipermetropía supone categorizaciones informativas que derivarán en el utilitarismo de los recursos y servicios hídricos, entonces el hedonismo no solo está desvinculado de los riesgos percibidos en el pasado y que no tendrían una repercusión significativa en el presente, sino además estaría relacionado con el utilitarismo que considera a los recursos naturales y servicios públicos como instrumentos de confort.

Utilitarismo y hedonismo, en tanto normas y valores socialmente diseminados en grupos que consideran al agua como un medio de confort, determinarían comportamientos antiecológicos. El estudio de Frías et al. (2009) demuestra que las normas sociales cristalizadas en principios individuales determinan acciones específicas, pero en tanto se materializan en estándares morales definen una identidad en función del contexto. Esto es, que la hipermetropía también es resultado de un proceso de identificación del individuo con respecto a las conductas de un grupo y las acciones sociales ante sequías o inundaciones, que fueron diseminadas en los medios de comunicación y que propiciaron una respuesta hedonista más que conservacionista. 
La hipermetropía estaría reflejada por el utilitarismo y el hedonismo que el grupo de referencia o pertenencia desarrolló e influyó sobre el individuo en un entorno hostil, aunque permisible con grupos que sesgaron sus percepciones hacia sus capacidades de autogestión de recursos naturales y servicios públicos.

Sin embargo, desde la perspectiva de Gilford (2009), el pesimismo más que el fatalismo es diferente por su nivel espacial: local, nacional y global. En consecuencia, la hipermetropía no solo es un sesgo perceptual de las normas sociales, grupales y personales indicadas por su grado de utilidad y hedonismo, sino además es un sesgo de escenarios que el perceptor desconoce y por ende homogeniza para tener un control o certidumbre del contexto de disponibilidad hídrica.

La hipermetropía, como un sesgo espacial, explicaría la biofilia en contextos de diversidad natural como determinante del comportamiento proambiental. Corral et al. (2009) modelaron ambas variables con la intolerancia social y la edad para demostrar que existía una relación implícita entre la conservación del entorno y la afinidad hacia la naturaleza. En este sentido, la hipermetropía estaría vinculada con la intolerancia social ya que la biofilia supondría acciones inmediatas y específicas de conservación del entorno inmediato, aunque una vez garantizada la existencia de especies, el individuo podría desarrollar un hedonismo y utilitarismo hacia su entorno preservado.

En virtud de sus implicaciones normativas, valorativas, perceptuales, actitudinales y comportamentales la hipermetropía es un constructo psicológico sustentable complejo que supone un estudio interdisciplinar. Las implicaciones que el estudio de la hipermetropía tendría para las políticas ambientales y los servicios públicos son inéditas.

En principio, una hipermetropía sociopolítica estaría indicada por sesgos perceptuales en torno a la relación entre sociedad, Estado y naturaleza. En ese tenor, el utilitarismo y el hedonismo reportado en los estudios de la psicología de la sustentabilidad servirían para conceptualizar la dimensión sociopolítica de la hipermetropía como aquella en la que el cambio climático, la escasez de recursos y el desabastecimiento en las urbes sería un instrumento de poder y control que reduciría la participación civil a conflictos con sus autoridades y a los recursos naturales en servicios de tandeo.

De este modo, la hipermetropía sociopolítica explicaría las movilizaciones sociales y las acciones colectivas tales como manifestaciones, mítines, plantones o marchas como instrumentos de presión y negociación entre usuarios del servicio público y autoridades locales.

Precisamente, el objetivo del presente trabajo es especificar el constructo de hipermetropía sociopolítica para delinear su estudio en un modelo reflejante. Para tal propósito se realizó una investigación documental en las bases de datos de DIALNET, LATINDEX y REDALYC. Posteriormente, se procesaron las definiciones en una matriz de análisis de contenido y, por último, se modelaron los indicadores extraídos en la revisión del estado del conocimiento. 


\section{PROBLEMÁTICAS SOCIOPOLÍTICAS GLOBALES Y LOCALES EN TORNO AL ABASTECIMIENTO DE AGUA}

Desde el enfoque económico, la problemática del agua supone un desbalance entre disponibilidad y consumo que solo podrá ser resuelto si se implementa un sistema de tarifas.

En el mundo el $97,5 \%$ del agua es salada, $2,24 \%$ es dulce y solo $1 \%$ se encuentra disponible en ríos, lagos y acuíferos para el consumo humano. $113.000 \mathrm{~km}^{3}$ de agua se precipitan anualmente (United Nations Water, 2013, pp. 13-18).

La disponibilidad del recurso ha disminuido paulatinamente. En 1950 solo el continente asiático tenía una baja disponibilidad y para el año 2025 la escasez se extenderá a los cinco continentes. El desequilibrio entre la explotación (se estiman $4.600 \mathrm{~km}^{3}$ ) del recurso y su recarga natural afectará su disponibilidad para el consumo $\left(2.400 \mathrm{~km}^{3}\right.$ aproximadamente) en la agricultura, la industria y las actividades domésticas (United Nations Habitat, 2010, pp. 16-29).

En el caso de México, al ser el onceavo país con más población en el mundo (101,7 millones de personas), con una densidad de 52 personas por $\mathrm{km}^{2}$ en promedio; una población menor de 15 años (33\%), el $74 \%$ vive en zonas urbanas y su ingreso per cápita al año es de 8.790 dólares norteamericanos trabajando 40 horas a la semana, su crecimiento anual es de 2,1 millones y se espera que en el 2050 aumente $48 \%$, estimando su población en 131, 7 millones para el 2030, ha sido clasificado con un índice de disponibilidad extremadamente baja con menos de $1.000 \mathrm{~m}^{3}$ por habitante al año (Instituto Nacional de Estadística Geografía e Informática, 2010, pp. 3-24).

Respecto a las zonas centro y norte del país donde el crecimiento económico es significativo, la disponibilidad del recurso ha sido clasificada como muy baja con 1.000 a $2.000 \mathrm{~m}^{3}$ por habitante al año. Solo el sureste de México que ha tenido un crecimiento económico poco significativo, ha sido clasificado con una alta disponibilidad de $10.000 \mathrm{~m}^{3}$ por persona al año. Las zonas norte, centro y noreste que contribuyen con el $85 \%$ del Producto Interno Bruto (PIB) y tienen el $77 \%$ de la población solo cuentan con el $32 \%$ de la disponibilidad de agua, aproximadamente $1.874 \mathrm{~m}^{3}$ por habitante al año. En contraste, la zona sureste que contribuye con $15 \%$ del PIB y concentra el $23 \%$ de la población, tiene una alta disponibilidad del $66 \%$ de los recursos hidrológicos, aproximadamente $13.759 \mathrm{~m}^{3}$ por persona al año. De este modo, el promedio de disponibilidad de agua nacional es de $4.573 \mathrm{~m}^{3}$ por individuo al año (Comisión Nacional del Agua, 2012, pp. 14-16).

En el caso de la Zona Metropolitana del Valle de México (ZMVM) habitan 18'620.763 concentrados en un área de $4.979 \mathrm{~km}^{2}$ y una densidad poblacional de 3.740 personas por $\mathrm{km}^{2}$ agudizan el servicio intermitente de agua potable como la principal problemática. En la ZMVM, durante el período que va de 1950 al año 2000, la población ha aumentado 5,25 veces y pasó de 3'442.557 habitantes a 18'076.572 residentes. Respecto a la densidad poblacional el Estado de México y el Distrito Federal son la primer y segunda entidades con más viviendas ocupadas, siendo la delegación Iztapalapa la más poblada con 1'750.336 de los cuales la mitad son menores de 15 años (Comisión Nacional de Población, 2008, pp. 23$55)$. 
Tal panorama se exacerba si se considera que la calidad del agua superficial de 393 estaciones en 225 ríos, 81 estaciones en 62 lagos y presas, 26 estaciones en 13 santuarios y sitios costeros, 15 estaciones de descarga de aguas residuales ha sido reportada como muy baja, así como de la subterránea que consta de 228 estaciones en 24 acuíferos, establecidos por el Índice de Calidad del Agua con valores entre 0 y 100 , siendo este último valor excelente, después aceptable, levemente contaminada, contaminada, fuertemente contaminada, y el último como excesivamente contaminada. El $60,7 \%$ del agua superficial y el $46,3 \%$ de subterránea están contaminadas y fuertemente contaminadas, clasificando al agua superficial del Valle de México como excesivamente contaminada con un 32,49 (Comisión Nacional del Agua, 2005, p. 13).

El suministro de agua en la ZMVM es de $68 \mathrm{~m}^{3} / \mathrm{s}$. El cual proviene: de pozos explotados $25,16 \mathrm{~m}^{3} / \mathrm{s}(37 \%)$, pozos recargados $15 \mathrm{~m}^{3} / \mathrm{s}(22 \%)$, manantiales $0,36 \mathrm{~m}^{3} / \mathrm{s}(2 \%)$, del río Cutzamala $13,6 \mathrm{~m} / \mathrm{s}(20 \%)$, del río Lerma $6,12 \mathrm{~m}^{3} / \mathrm{s}(9 \%)$ y saneamiento con $6,8 \mathrm{~m}^{3} / \mathrm{s}(10 \%)$. Respecto al grado de presión del recurso, la ZMVM ocupa el primer lugar con una sobreexplotación del $120 \%$ del agua disponible. A pesar de que la situación hidrológica es extremadamente comprometida en la ZMVM, las demás regiones hidrológicas se encuentran muy cercanas a dicha problemática. Las zonas norte, noreste y centro explotan el $40 \%$ de sus recursos hidrológicos (Comisión Nacional del Agua, 2008, pp. 58-64).

En el caso de la Ciudad de México en 1955 sus habitantes tenían una disponibilidad de $11.500 \mathrm{~m}^{3}$ anuales per cápita. En el año 2004 disminuyó a $4.094 \mathrm{~m}^{3}$ anuales per cápita. En ese mismo año se consumió el $74 \%$ del total de agua potable suministrada equivalente a $16.157 \mathrm{~m}^{3} / \mathrm{s}$. El agua en el Distrito Federal, es destinada a la industria (17\%), el comercio (16\%) y uso doméstico (67\%) el cual se divide en el uso de excusado (40\%), regadera $(30 \%)$, ropa $(15 \%)$, trastes $(6 \%)$, cocina $(5 \%)$ y otros $(4 \%)$. Iztapalapa al concentrar la mayor población obtuvo el mayor consumo con $2.732 \mathrm{~m}^{3} / \mathrm{s}$ equivalentes al $16,9 \%$ del total. Gustavo A. Madero y Álvaro Obregón con $1,375 \%$ y 9,94\%, respectivamente. En contraste, las delegaciones con menor consumo fueron Cuajimalpa, Tláhuac y Milpa Alta con un 5,97\%. En este sentido, se espera para el 2020 una disponibilidad de $3.500 \mathrm{~m}^{3}$ anuales per cápita. Por ello la cobertura del servicio es excluyente con 905.000 personas que no cuentan con agua potable porque hay una escasez de $6 \mathrm{~m}^{3} / \mathrm{s}$ (Comisión Nacional del Agua, 2012, pp. 30-36).

En el caso del consumo doméstico de agua los criterios para establecer la escasez son:

- $\quad$ Crítica entre 1.000 y $1.700 \mathrm{~m}^{3}$ anuales per cápita.

- $\quad$ Baja entre 1.700 y $5.000 \mathrm{~m}^{3}$ anuales per cápita.

- $\quad$ Media entre 5.000 y $10.000 \mathrm{~m}^{3}$ anuales per cápita.

- $\quad$ Alta más de $10.000 \mathrm{~m}^{3}$ anuales per cápita.

El $32,27 \%$ de los usuarios se encuentra dentro del umbral del rango, el $78,5 \%$ tiene un consumo menor a $50 \mathrm{~m}^{3}$, el $11 \%$ consume menos de 10 $\mathrm{m}^{3}$ y 0,38 consume más de $180 \mathrm{~m}^{3}$ bimestrales. El pago promedio en la Ciudad de México es de 110,25 pesos bimestrales. Esto significa una recaudación por cobro de derechos a los usuarios del $80 \%$ en relación a su costo real por el servicio (Comisión Nacional del Agua, 2008, p. 31). 
Se estima que en el año 2025 el 80\% de la población mundial estará en alta escasez. El pronóstico para el año 2050 implica un rango de aumento de la temperatura de 1,4 a $5,60 \mathrm{C}$ causando un incremento de $44 \mathrm{~cm}$ del nivel del mar, un $5 \%$ más de las precipitaciones y la extinción de una cuarta parte de las especies. En este sentido, se estima para el año 2025 una crisis mundial de abasto irregular e insalubre de agua en la que 2.000 millones de individuos no dispondrán de agua bebible. En el año 2030 el crecimiento poblacional de la ZMVM será de 22,5 millones de habitantes, la Zona Metropolitana de Guadalajara (ZMG) de 4,8 millones de habitantes y la Zona Metropolitana de Monterrey (ZMM) de 4,9 millones de habitantes. La problemática de distribución de los recursos hídricos se enfocaría en aquellas ciudades con una población mayor a los 500.000 habitantes. Si se consideran las proyecciones poblacionales, las zonas metropolitanas de Guadalajara, Monterrey, Cuernavaca, Tlaxcala, Veracruz, Puebla, Aguascalientes, Toluca, San Luis y Cancún estarían en una crisis de disponibilidad hídrica esperada para el año 2025 (Comisión Nacional del Agua, 2008, pp. 12-15).

El enfoque económico (García y Bustos, 2013) ha establecido un sistema de cobro para reducir el desbalance entre disponibilidad hídrica y consumo de agua, en consecuencia:

Tarifa por estándar. La tasa por unidad de agua es independiente de la cantidad de agua consumida. La tarifa es la misma sin importar la disponibilidad o el consumo de agua.

Tarifa por volumen. El precio unitario del agua depende de la cantidad que se utiliza, empero se incrementa o disminuye a partir de la discrecionalidad gubernamental.

Tarifa por situación. La tasa por unidad de agua aumenta su costo durante el día y disminuye su costo durante la noche. Durante la temporada de estío se incrementa su costo y durante la temporada de lluvias disminuye su precio unitario.

Tarifa por intervalos. El precio unitario del agua se incrementa en función del volumen consumido. A partir de los intervalos de consumo se aplican precios que se incrementan conforme el consumo sobrepasa los umbrales permitidos.

Tarifas por umbrales. El precio unitario del agua es constante en tanto no rebase el umbral de confort. Una vez rebasado el consumo asignado, se aplica un incremento logarítmico.

Tarifa por autofinanciamiento. El costo unitario del servicio se establece a partir del ingreso familiar y un umbral de confort. Una vez rebasado el límite permitido, se incrementa el costo por cada volumen cúbico extra.

Tarifa por subsidio. El costo unitario del servicio de agua potable implica una cuota estándar o estratificada y un subsidio en función de un umbral de confort.

El enfoque económico sostiene que la disponibilidad de recursos es un indicador de la huella ecológica, la cual puede ser reducida a partir de un balance de costos y beneficios. A medida que los recursos naturales escasean, el incremento de tarifas correspondientes orientaría el consumo de otros recursos con mayor disponibilidad. Mientras tanto, el recurso 
natural en vías de extinción podría conservarse ya que estaría protegido por el alto costo que supone su consumo, empero el consumo de un recurso, desde la aproximación psicosocial, es determinado por procesos de influencia social. Cuando menos, la psicología de la sustentabilidad ha establecido dos procesos de influencia, uno de orden mayoritario o directo y otro minoritario o indirecto, relativos a la hipermetropía.

\section{ESPECIFICACIÓN SOCIOPOLÍTICA DE LA HIPERMETROPÍA}

La especificación sociopolítica de la hipermetropía supone el establecimiento de los efectos de las campañas ecocéntricas en la preservación del mercado hidrológico. A partir de la década de los sesenta, se inician los movimientos ecologistas que demostrarán los efectos nocivos de la economía de mercado, las políticas liberales, las sociedades industriales, la masificación de los servicios y el consumismo de productos diversificados (Amérigo et al., 2005). Posteriormente, en la década de los setenta, surgen los movimientos antiarmamentistas que se preocupan más por la preservación de las especies animales y vegetales (Larson et al., 2009).

Las movilizaciones conservacionistas planteaban la explotación de los recursos en función de su disponibilidad. En la década de los ochenta, las ideas y las acciones ambientalistas tales como: boicots de productos y servicios, dosificación del consumo e incluso el abstencionismo, caracterizan las sociedades postindustriales. Ante la incertidumbre e inseguridad derivadas de las explosiones radioactivas del reactor de Chernobyl, los grupos ambientalistas se organizaron para protestar masiva y sistemáticamente en las principales ciudades del mundo. La caída del bloque socialista evidenció nuevas formas de destrucción nuclear del medio ambiente y, con ellas, nuevas formas de organización ambientalista (Lucio y Pinheiro, 2013).

Las marchas, los mítines y las manifestaciones dieron paso a las demostraciones realistas del exterminio de las especies cuando grupos ambientalistas trasladaban cetáceos muertos a las plazas de las ciudades europeas. Estas demostraciones se complementaron con acciones de intervención directa para evitar el exterminio de ballenas, focas, osos o aves. Las manifestaciones dejan las calles y entran a los portales de las instituciones gubernamentales (Milfont et al., 2010). El bloqueo de servidores y el ataque a redes con virus informáticos son ejemplos del activismo que caracteriza a la década de los noventa. Finalmente, la consolidación del desarrollo sostenible extendió a los ámbitos económicos, políticos, sociales, culturales, educativos, científicos y tecnológicos el crecimiento en función de la disponibilidad de recursos sin afectar las capacidades de las generaciones futuras de utilizar dichos recursos. En este sentido, las campañas políticas han utilizado los principios del desarrollo sostenible para captar adeptos (Vervoort et al., 2014).

En el contexto hidrológico, se intercambian votos por la redistribución del agua. Sin embargo, el desarrollo sostenible coexiste con otra forma de desarrollo hedonista, improvisado y heurístico: el consumo líquido (García et al., 2013).

La influencia mayoritaria plantea que el consumo sistemático de un recurso es determinado por el poder de decisión de la mayoría. Si el grueso de la población tiene por costumbre el aseo personal diario, entonces el individuo se verá influido a adoptar un estilo de vida 
antropocéntrico en el que los recursos hídricos son considerados como un servicio exclusivo para las necesidades humanas actuales, sin importar las capacidades de las generaciones humanas posteriores así como las necesidades de las especies actuales o futuras (Basar, 2011). El modelo mayoritario es directo porque a través de una fuente considerada experta puede influir sobre la decisión de consumo del individuo. En efecto, la conformidad del individuo es el resultado final de la influencia mayoritaria (Real y García, 2001).

En contraste, la influencia minoritaria sostiene que el consumo de los recursos naturales obedece a la identidad que establece el individuo para con los grupos que le rodean. De este modo, el aseo personal puede variar según el estilo de vida del grupo al que el individuo pertenece. Si el grupo tiene por norma el aseo personal con un mínimo de agua, entonces el individuo llevará a cabo dicha acción sin importar la disponibilidad de agua (Moser y Uzzell, 2004). Se trata de una influencia indirecta ya que impacta el estilo de vida futuro más que la decisión de consumo en el presente. Por ello, la innovación es la consecuencia principal de la influencia minoritaria.

Ambos procesos de influencia social, mayoritario o minoritario, parecen obviar la disponibilidad de los recursos que el enfoque económico muestra como factor esencial, empero son relevantes porque advierten que sin importar la cantidad de agua consumible, la toma de decisión presente o futura es determinada por la norma social o por la norma grupal (García et al., 2005).

Los símbolos, los significados y los sentidos que le corresponden son los medios que implican a las culturas prematerialistas, las culturas materialistas y a las culturas postmaterialistas con el entorno ambiental (Uzzell et al., 1994). En las culturas premateriaistas, la naturaleza es simbolizada como un conglomerado de elementos comunitarios tan significativos como los elementos humanos que forman un grupo (Pol y Castrechini, 2013).

En contraste, a menudo, la naturaleza es simbolizada como recursos inagotables por parte de los grupos que la transforman y la redistribuyen propiciando las desigualdades características de las sociedades neoliberales económicas. Finalmente, cuando las culturas postmaterialistas han alcanzado un estatus económico y educativo muy alto, la naturaleza es simbolizada como un escenario de los derechos de cada organismo por su subsistencia (Rathzel y Uzzell, 2012). A partir de estas distinciones socioculturales, nueve teorías explican las cosmovisiones culturales de la naturaleza.

\section{DISCUSIÓN}

La Psicología de la Sustentabilidad (PS) ha establecido marcos teóricos y conceptuales para explicar la relación causal entre disponibilidad de agua y consumo per cápita a través de procesos cognitivos. En este sentido, el objetivo del presente trabajo es exponer las teorías en torno a las situaciones ambientales. Para tal propósito, a partir de una revisión del estado del conocimiento se explican las relaciones causales entre la exclusión hidrológica y la cultura, el Estado, la sociedad, los medios, las 
comunidades, el barrio, las familias y los individuos. Se plantean modelos teóricos para la explicación de las creencias, los valores, las percepciones, las actitudes, los conocimientos, las motivaciones, las habilidades, las intenciones y los comportamientos en función de la escasez, desabasto e insalubridad ambientales (Tronu, 2011). La exposición del marco teórico referencial servirá para abrir la discusión en torno a la precisión, evolución, construcción e innovación de modelos teóricos que enlacen los ámbitos personales, familiares, territoriales, locales, regionales y globales en los que la escasez de agua impacta al comportamiento humano mediante la regulación de procesos sociocognitivos básicos.

Se exponen las tendencias situacionales hídricas globales hasta locales para proyectar las sustentabilidades hídricas correspondientes. En este sentido, las políticas públicas son esenciales para establecer los costos de la oferta hídrica. Sin embargo, la participación ciudadana, comunitaria y barrial también es fundamental en el establecimiento de precios unitarios de agua.

De este modo, en el ámbito global, la sustentabilidad hídrica es determinada por políticas públicas que incentivan el ahorro de agua a través de estándares internacionales tarifarios. El precio del agua sería una consecuencia de convenios internacionales en los que los países firmantes se comprometen a disminuir sus procesos agrícolas, industriales y comerciales. El costo unitario estaría definido por el nivel de disponibilidad per cápita. Una mayor cantidad de agua para cada persona implicaría un costo estándar para demandante (Uzzel, 2000). Un consumo superior a un umbral incrementaría exponencialmente el precio unitario. A nivel global, los costos se reducirían y los beneficios se incrementarían sustancialmente. Sin embargo, los presidentes o ministros no pueden tomar decisiones globales sin comprometer el desarrollo local.

En este sentido, en el nivel continental, la relación entre el Norte industrial y el Sur agrícola, el comercio entre los bloques económicos, incide directamente en los flujos financieros y migratorios que deben ser considerados en la ecuación de una política pública para la sustentabilidad hídrica. En el ámbito de los continentes, el establecimiento de un sistema de cobro del servicio de agua es más factible si se consideran los acuerdos comerciales entre los integrantes de los bloques económicos. En tanto grupo geopolítico, una política pública de tarifas estaría orientada al subsidio de los ciudadanos ricos para la subsistencia de los residentes pobres (Uzzel, 2004). Se trata de un sistema tarifario en el que quienes tienen un mayor poder adquisitivo pagan una tarifa estándar que incluye el financiamiento de aquellos que viven en la exclusión, marginalidad o vulnerabilidad. No obstante, los localismos siguen teniendo un peso específico al momento de establecer las tarifas estándar y, por ello, se requiere revisar el ámbito nacional para esclarecer los factores que impiden la sustentabilidad a nivel continental.

Una causa de los bloques económicos han sido los nacionalismos. Desde los conflictos nacionalistas que llevaron a Europa a crear un mercado común, hasta los regionalismos que hoy promueven el derrumbe de monarquías en África y Medio Oriente, las ideologías locales han determinado el devenir de las sociedades. En México, los conflictos por el derecho al agua han sido paliados con políticas públicas centralistas y federalistas en las que se justifica la extracción y distribución de una cuenca a otra. El Estado, a través de las estimaciones de la Secretaría de Hacienda y Crédito Público (SHCP), el Banco de México (BM) y la 
Comisión Nacional de Agua (Conagua), ha establecido las políticas públicas orientadas al crecimiento económico más que al desarrollo sustentable. El Estado ha soslayado los derechos y patrimonios hídricos de las comunidades rurales y los barrios urbanos. En tal sentido, cada unidad de agua tiene un precio diferente e inequitativo. El agua es barata para quienes tienen mayor poder adquisitivo y consumen más. En cambio los grupos que ahorran agua, a pesar de estar desempleados o subempleados, pagan cinco veces más su costo real. Sin embargo, la política hídrica nacional es diversificada a nivel estatal.

En un país federalista, los gobiernos estatales son un contrapeso a la omnipresencia del ejecutivo. Una iniciativa del presidente puede ser modificada por la cámara alta y baja, el congreso estatal y el gobernador. Si a esto se agrega la altitud en la que viven las comunidades rurales y los barrios urbanos, tenemos que los estados legislarían un sistema de tarifas diferencial para cada entidad. Por ello, la sustentabilidad hídrica estatal tendría en las finanzas de los estados su principal obstáculo. A menudo, los gobiernos estatales gastan más de lo que reciben por parte de la federación. Esto propicia que los organismos operadores nacionales y locales busquen acuerdos para construir un sistema de subvención que beneficie con bajos precios a los usuarios. El resultado, es una acción pública organizada para el cobro pero desorganizada para el suministro. Sin falta, los recibos son distribuidos a los usuarios pero el servicio de agua es intermitente. Por ello, la sustentabilidad hídrica estatal beneficia a las urbes en detrimento de las zonas rurales.

La ZMVM es un caso paradigmático de la tendencia hídrica en la historia de la humanidad. Desde siempre, la ZMVM ha sido depositaria de precipitaciones, filtraciones y descargas de ríos y lagunas. Podría asegurarse que desde la fundación del Valle de México había una evidente sobrecarga. Al pasar los siglos y las culturas la cuenca se ha ido vaciando. Se estima que la tendencia de extracción actual alcanzaría para unas dos o tres décadas hasta comprometer su estructura. La complejidad geográfica no es menor a la complejidad sociopolítica. El Valle de México, administrado por tres entidades con sus respectivos congresos, incluye una política hídrica metropolitana que estaría definida por representantes de diferentes localidades con diferentes necesidades, expectativas y capacidades de consumo. No obstante, el Sistema Político Mexicano (SPM) se caracteriza por homogeneizar las demandas de la gente y las ofertas correspondientes. A partir de este rasgo político estructural, una política hídrica de sistema de precios es en realidad un sistema de concesiones, subsidios y condonaciones. Se trata de una política pública que no necesita ser legislada para ser implementada. En tiempos electorales, la eficacia del sistema político utiliza al servicio de agua potable como su instrumento de promoción, definición y elección de candidatos y representantes. De tal manera, la sustentabilidad hídrica metropolitana es discrecional, proselitista y clientelar. Consecuentemente, a nivel delegacional, la corrupción, el nepotismo y el clientelismo son sus componentes principales.

Una consecuencia de la complejidad del Valle de México son sus demarcaciones delegacionales y municipales. La diversidad de factores que influyen en la sustentabilidad hídrica metropolitana también incide en la sustentabilidad hídrica a nivel delegacional. Sin embargo, las delegaciones se agrupan en dos grupos: incluyentes y excluyentes. Las primeras presentan una densidad poblacional baja y altos ingresos que permitirían solventar un incremento exponencial de las tarifas. Las 
segundas presentan hacinamiento, desempleo o subempleo con ingresos insuficientes para costear una variación mínima del precio unitario del agua. En el caso de la delegación Iztapalapa, habría que añadir la altitud y la corrupción en la que viven los barrios con mayor desabasto e insalubridad. Se trata de una delegación en la que convergen diversos factores, y acerca a una crisis hídrica a sus pobladores. Tal situación propicia el surgimiento del emplazamiento o intenciones-acciones de inconformidad, protesta, confrontación o boicot encaminados a obtener una mayor cantidad de agua. Es así como la sustentabilidad hídrica en Iztapalapa requeriría de tarifas ajustadas a los usos y costumbres de sus pobladores que combaten la corrupción pero al mismo tiempo aceptan el clientelismo. En este sentido, los estilos de vida familiares-residenciales complementan sus movilizaciones colectivas.

En el ámbito residencial, el desabasto de agua es la tendencia principal que llevaría a las familias al acaparamiento, la dosificación y la seudorreparación de fugas. En efecto, un sistema de tarifas ajustado a las estrategias de austeridad implicaría umbrales de consumo determinados por el número de residentes, sus actividades económicas y tipos de esparcimiento. En el nivel de los grupos familiares-residenciales la sustentabilidad hídrica significa un pago subvencionado a quienes ahorran agua y un precio exponencial a quienes la derrochan o acaparan. Las habilidades de consumo y seudorreparación de fugas implicarían una subvención menor que se ajustaría a un precio estándar considerando la tendencia a futuro.

Finalmente, las tendencias hídricas expuestas proyectan escenarios futuros en los que la densidad urbana es un problema global, nacional y local que afecta la sustentabilidad hídrica. Es decir, la disponibilidad per cápita esperada para los próximos años es una consecuencia de las políticas públicas que buscan frenar la tendencia hídrica más que hacerla sustentable. En ese sentido, es predecible la desaparición de sistemas estándares, subsidiados, situacionales, intervalares o umbrales de tarifas. En su lugar, un nuevo sistema de precios deberá implementarse para subsanar las fallas estructurales. Se trata de un sistema de tarifas determinado por las contingencias hídricas globales hasta locales.

En torno a las problemáticas ambientales las ciencias sociales plantean los siguientes enfoques:

Estudios Regionales-Comunitarios. Las problemáticas hídricas, relacionadas con la solidaridad en tiempos de escasez y con la festividad en tiempos de abundancia, son sentidas como elementos de la comunidad. La autogestión de la comunidad es la manifestación principal ante el desequilibrio hídrico global con efectos de escasez locales. Es decir, los lagos, ríos, lagunas, acuíferos o glaciares que históricamente pertenecen a las comunidades étnicas, al ser sobreexplotados por las urbes propician las movilizaciones de defensa del patrimonio comunitario por su preservación. En tal sentido, el Desarrollo Sustentable es equiparable a la autogestión de las comunidades por su derecho histórico del agua como su patrimonio de subsistencia.

Estudios Jurídicos. La principal problemática es la defensa de los derechos al acceso y consumo de agua. Es decir, los acuíferos, lagos y ríos que pertenecieron a los grupos étnicos fueron expropiados por sus gobiernos y redistribuidos a zonas de desarrollo económico industrial o agropecuario. De nuevo, el Desarrollo Sustentable es la solución a la 
problemática del reconocimiento jurídico de los pueblos a su autodeterminación. El Desarrollo Sustentable es concebido como un documento, tratado o acuerdo en el que se plasman las leyes o principios de la autonomía relativa de los pueblos, grupos o asentamientos humanos. Las instituciones tales como las secretarías de medio ambiente o las comisiones de derechos humanos, fungen como celosos vigías de los acuerdos que han firmado los estados para controlar sus abusos sobre las comunidades y los recursos que les corresponden.

Estudios Económicos-Sociales. Se estudian las problemáticas hídricas en relación a los índices de desarrollo humano. El desarrollo humano ideal está relacionado con la optimización de los recursos hídricos. En contraste, el desarrollo humano paupérrimo está vinculado con la escasez, corrupción, fugas y dispendio. Desde las ciencias económicas se plantea el dilema de la capitalización de los recursos naturales versus su conservación. Ante tal dilema, se propone el Desarrollo Sustentable en lugar del crecimiento económico y la preservación del entorno. Los planteamientos económicos hídricos establecen mecanismos de ahorro de agua a partir de sistemas tarifarios. El precio del servicio de agua potable se establece mediante las tasas de minimización o maximización de las relaciones entre los servicios, sus costos y sus beneficios en situaciones de intercambio. En este sentido, un incremento en los precios unitarios de agua incide en la reducción del consumo y la equidad distributiva. Precisamente, en las ZUP se establecen subsidios mientras que en la ZUC incentivos para la optimización y tratamiento y reutilización del agua. Los organismos económicos como el Fondo Monetario Internacional o el Banco Mundial, miden el desarrollo sustentable a partir de índices especializados en establecer la relación causal entre el ingreso per cápita y la salud, trabajo, educación, alimentación, calidad de vida o bienestar subjetivo.

Estudios Políticos-Sociales. El impacto de la escasez de los recursos hídricos sobre las zonas centrales y periféricas de las decisiones de inversión pública, es la problemática que enmarca el estudio de los conflictos entre la ciudadanía y sus autoridades. Son dos las problemáticas hídricas: la equidad y el financiamiento del servicio. Ante las demandas de la ciudadanía, los gobernantes ofrecen una mayor cobertura sobreexplotando los acuíferos y filtrando las aguas residuales. Se trata de una política pública orientada por una planificación sustentable; integral, eficiente, equitativa e incluyente. Las demandas por el recurso hídrico se manifiestan en plantones, mítines, marchas, propaganda y confrontaciones con la policía. Las movilizaciones ciudadanas son analizadas como "clientelas", un mecanismo de control político electoral de los partidos sobre los grupos excluidos. Ante tales problemáticas, se estudian las soluciones que giran en torno a una reforma electoral en la que los gobernantes ofrecen una mayor transparencia en el manejo de los recursos a cambio de una mayor participación ciudadana en las elecciones y la rendición de cuentas. En este sentido, se estudia al poder legislativo y sus iniciativas que permitan la participación directa de las mayorías y sobre todo las minorías, principales víctimas del crecimiento económico a costa de la sobreexplotación y escasez de recursos, en las decisiones de inversión y financiamiento para el desarrollo personal y grupal. El Desarrollo Sustentable, es el producto de debates entre la ciudadanía y el Estado, se trata de un acuerdo en el que ambas figuras políticas convienen la explotación racional, planificada y moderada de los recursos hídricos. 
Estudios Sociológicos. Las problemáticas hídricas se enfocan en la incertidumbre ambiental. Se considera al medio ambiente como un conjunto de variables inconmensurables, impredecibles e incontrolables que exhibe a la humanidad y a sus sociedades como parte del proceso de evolución y transformación de la naturaleza. Es decir, la naturaleza ha pasado por diferentes estadios evolutivos y el cambio climático que hoy en día se vive y sufre, es solo una etapa más del desarrollo de la Tierra. En dicha etapa los humanos se extinguirán salvo que sus sistemas puedan retardar los efectos del cambio climático o adaptar a sus descendientes a la contingencia ambiental. Las ciencias sociológicas plantean sociedades del riesgo en las que los avances tecnológicos tales como las centrales nucleoeléctricas, el transporte aéreo o la infraestructura hídrica en cualquier momento pueden colapsarse y con ello comprometer el crecimiento de las generaciones actuales y futuras. El Desarrollo Sustentable es un contexto de certidumbre en tiempos de riesgo, un contexto de seguridad en tiempos de incertidumbre, un contexto de confianza en tiempos de negligencia y corrupción.

Estudios Psicológicos. Las problemáticas hídricas consisten en el impacto de la disponibilidad del agua sobre las percepciones, emociones, actitudes, motivos, intenciones, habilidades, competencias y comportamientos. La escasez, desabasto, acaparamiento o insalubridad tienen un efecto directo sobre el ahorro de agua. Dicho efecto también es mediado por las variables cognitivas. Los psicólogos llevan a cabo la confiabilidad y la validez de los instrumentos que miden dichas variables para relacionarlas con otras variables situacionales, demográficas, educativas o geoespaciales. El Desarrollo Sustentable, consiste en la adopción de estilos cognitivos y de comportamiento anti o proambientales.

Las políticas públicas determinan las sustentabilidades hídricas globales, continentales, nacionales, estatales, metropolitanas, delegacionales, residenciales y prospectivas. Las políticas públicas tratan de organizar los factores determinantes de la disponibilidad hídrica a partir de parámetros tarifarios para el financiamiento de la sustentabilidad hídrica. Un incremento en el precio unitario del servicio de agua impacta directa, positiva y significativamente en su optimización y ahorro.

Las situaciones ambientales han sido planteadas desde los ámbitos demográficos (densidad poblacional austera), históricos (normas y valores de ahorro o dispendio), económicos (competencias por los recursos hidrológicos escasos), políticos (representatividad de las demandas ciudadanas), estructurales (capitales de dispendio), culturales (creencias de la disponibilidad), cognitivos (conciencia ambiental), mediáticos (opinión mediatizada) y comunitarios (identidad y apropiación espacial).

El ámbito demográfico. Enmarca la densidad poblacional (número de consumidores o demandantes de agua en una casa habitación) determinada por la disponibilidad del agua (cantidad de litros almacenados para las necesidades domésticas, higiénicas y de consumo). En efecto, se considera que en la relación naturaleza-humanidad, el agua es el principal recurso intermediario porque a partir de ella se desarrolló la agricultura que permitiría establecer los ciclos de producción en función de la temporada de precipitaciones. La programación de la siembra y la cosecha permitió el desarrollo de los pueblos. Sin embargo, debido a que la población en México tiende a concentrarse en zonas con una baja disponibilidad del recurso, es necesario complementar este ámbito 
demográfico con el ámbito histórico que nos permita responder a esa paradoja.

El ámbito histórico. Enmarca la difusión de valores y normas con respecto a la disponibilidad hidrológica. Se considera que en la relación naturalezahumanidad se han desarrollado valores biosféricos (mistificación, exploración y cuidado del entorno) y valores capitalistas (desmistificación, explotación y destrucción del entorno) que orientan normas al interior de un grupo, después se amplifican a una comunidad, se extienden a una región $y$, finalmente, se expanden a escala mundial a través de comportamientos deliberados e irracionales, planificados y heurísticos, sistemáticos e improvisados. A partir de tales procesos, la humanidad compite por los recursos y al mismo instante se solidariza para redistribuir dichos recursos. En el caso del agua, la humanidad la ha preservado con hábitos de dosificación, ahorro y reutilización que, sistemática e improvisadamente, agotan al recurso comprometiendo su uso en el futuro. El ámbito histórico enmarca los procesos valorativos en torno al recurso hidrológico, tiene su complemento en el ámbito económico que enmarca la competencia por el abasto regular y salubre del agua.

El ámbito económico. Enmarca la relación naturaleza-humanidad como procesos asimétricos en los que la humanidad compite por los recursos hasta comprometer su renovación, provocar su extinción u obligar a su transformación. En el caso de los recursos hidrológicos, la humanidad no solo los ha sobreexplotado, sino además los ha sobreutilizado. Por ejemplo, los ríos más importantes para los ecosistemas (Amazonas, Plata, Ganges, Yangtsé, Nilo, Mississipi) han sido utilizados como generadores de energía al instalarse hidroeléctricas en sus afluentes con el propósito de garantizar el abasto energético a las ciudades. Ante tales escenarios, ha predominado el enfoque neoclásico que considera a los recursos naturales como bienes externos a las esferas públicas y privadas, justificando con ello, su sobreexplotación por parte del capital privado. Sin embargo, el enfoque de la economía ecológica busca contrarrestar este planteamiento al considerar que los recursos son bienes prospectivos cuya explotación irresponsable en el presente compromete su disponibilidad en el futuro afectando a las generaciones subsecuentes. Este planteamiento de la economía ecológica se complementa con el ámbito político porque implica al Estado como el principal actor que atenderá las demandas hidrológicas de la ciudadanía y gestionará la inversión o promoverá la educación de dicha ciudadanía en función de la disponibilidad hidrológica.

El ámbito político. Enmarca la relación naturaleza-humanidad, se encuentra el Estado como regulador de dicha relación. En tal sentido, el Estado no solo es un ente que recibe solicitudes de abastecimiento de agua, orienta la inversión pública y redistribuye los recursos hidrológicos, sino también es un escenario de debate de demandas y gestiones. En dicho escenario, la relación naturaleza-humanidad ha sido sustituida por tres relaciones:

Infraestructura-contribuyentes. El Estado argumenta que en la solución a las situaciones de escasez, abasto irregular e insalubridad del agua, es imprescindible la inversión pública. El Estado gestiona los préstamos en organismos internacionales financieros para construir la infraestructura orientada a la distribución, medición, cobro y multas por los servicios de agua potable, drenaje y alcantarillado. 
Agua-ciudadanía. El Estado promueve el cuidado del agua en escuelas de nivel básico, medios de comunicación, vías de transporte y espacios públicos con la intención de concientizar a la ciudadanía en torno al pago de sus impuestos, no en función de la disponibilidad hidrológica sino en función de la disponibilidad de los recursos públicos.

Epidemia-comunidad. El Estado diseña, legisla e implementa programas de asistencia sanitaria a las comunidades excluidas del agua salubre o en las zonas vulnerables donde se desatan epidemias gastrointestinales. A través de trabajadores sociales y estadísticas de centros de salud, recopila información sobre la salud de las comunidades y el servicio de agua potable.

Las tres relaciones suponen un Estado benefactor que trata de regular la relación naturaleza-humanidad a partir de la administración de recursos, humanos, financieros e hidrológicos. Precisamente, el ámbito estructural se enfocará en estos recursos conceptualizándolos como capitales; humanos, financieros y naturales.

El ámbito estructural. Enmarca a los individuos que al interactuar construyen sistemas sociales a partir de sus recursos económicos y educativos. De este modo, las personas se encuentran ubicadas en posiciones sociales desde las cuales sistematizan o improvisan comportamientos, que las identifican como un grupo homogéneo. Las investigaciones desde este ámbito han encontrado que los hombres, profesionistas y con ingresos altos son más proclives a reconocer y cuidar el medio ambiente, así como a financiar movimientos ecologistas. Este prototipo de ser humano se ha identificado como ambientalista. Sin embargo, los indicadores del ambientalismo no son suficientes para explicar el significado del Desarrollo Sostenible. Por ello, el ámbito cultural ha desarrollado una explicación más atingente.

El ámbito cultural. Enmarca a los ambientalistas que en culturas anglosajonas más que en culturas latinas, tienen visiones del mundo postmaterialistas (cuestionamiento al progreso económico) y ecocéntricos (preservación ambiental más que crecimiento económico). En contraste, existen personas con bajos ingresos, concentradas en la periferia de las urbes, con niveles educativos bajos, quienes tienen visiones del mundo materialistas (crecimiento económico) y antropocéntricas (se considera que la naturaleza debe satisfacer solo las necesidades humanas). Debido a que el Desarrollo Sostenible sugiere garantizar los derechos y capacidades de las futuras generaciones para desarrollarse, el ámbito cultural explica el origen de la controversia entre quienes están a favor y en contra de la sostenibilidad. Sin embargo, en la humanidad, la ciudadanía e incluso en las comunidades, existen procesos deliberados, planificados y sistemáticos a partir de los cuales los individuos subyacen e incluso sustituyen a los grupos. Se trata de un proceso que el ámbito cognitivo ha enmarcado fehacientemente.

El ámbito cognitivo. Enmarca la función mediadora de las percepciones, actitudes, motivos, conocimientos e intenciones. Esto implica que la escasez de recursos naturales afecta indirecta, positiva y significativamente al comportamiento humano, más aún, dicho efecto está mediado por factores cognitivos que al interactuar configuran procesos planificados. El ámbito cognitivo ha demostrado que los motivos, la percepción de control y de riesgos, los conocimientos, las actitudes y la intención han sido variables mediadoras o transmisoras de los efectos de 
una situación de escasez sobre el comportamiento humano. Sin embargo, el comportamiento ecológico ha sido contradictorio al existir una baja correlación entre el reciclaje y la reutilización en culturas latinas. En consecuencia, se ha planteado al Desarrollo Sostenible como la solución a la problemática. En esencia, este planteamiento sugiere un comportamiento que beneficie tanto a las generaciones presentes como a las futuras, a través de acciones tales como el control de fugas y el ahorro de agua. El ámbito cognitivo, solo enmarca el impacto de la escasez de recursos naturales sobre el comportamiento humano planificado. Es en el ámbito mediático el que se enmarca el impacto de la situación del agua a través de la difusión masiva de la situación hidrológica.

El ámbito mediático. Enmarca la apropiación de mensajes en torno a la situación ambiental y las acciones gubernamentales. Los medios de comunicación al difundir la situación hidrológica y las acciones gubernamentales en torno a la problemática, propician un efecto directo y significativo en las decisiones y acciones ciudadanas. La opinión pública, al ser influida por la información mediatizada, construye decisiones que la llevan a participar a favor o en contra de las políticas públicas hidrológicas. Las acciones gubernamentales tienden a responder más a las demandas de los medios que a las demandas ciudadanas. De este modo, las manifestaciones, bloqueos y confrontaciones con la autoridad son explicados a partir del estudio de los barrios considerados como comunidades.

El ámbito comunitario. Enmarca la construcción social del sentido de pertenencia hacia un barrio y el apropiamiento de los espacios tales como: las calles, los parques, las plazas y las escuelas a partir de la memoria colectiva (construcción de imágenes y discursos en torno a un proceso o entidad). La realidad, cualquiera que ésta sea, implica a los sistemas identitarios locales y los sistemas económicos globales mutuamente incluyentes pero también mutuamente excluyentes. Principalmente, cuando los productores y los consumidores demandan nuevas relaciones a partir de los recursos sobreexplotados, la producción innovadora, el crédito diversificado, el consumo inusitado y la ecología deteriorada. Por lo tanto, el ámbito comunitario enmarca el impacto del dispendio hidrológico de las zonas económicas opulentas sobre las necesidades de abasto de las zonas económicas excluidas. Plantea el cambio social a partir del conflicto entre la estructura económico política y las minorías demandantes.

Los ocho ámbitos enmarcan situaciones derivadas por la distribución excluyente de recursos hidrológicos y sociales sean económicos, políticos o psicológicos. La exploración de las situaciones es enfocada en la distribución preliminar de los recursos. La descripción de las situaciones es delimitada a los indicadores de abundancia o escasez de los recursos. La explicación es concentrada en las causas y efectos de la distribución de los recursos. Finalmente, la comprensión es construida a partir de los símbolos, los significados y los sentidos que se vinculan con los recursos. Se recuperan las unidades de análisis, los métodos de estudio y las técnicas de investigación de las ocho aproximaciones. Sin embargo, los ámbitos solo enmarcan la problemática de distribución de los recursos en alguno de los elementos del sistema. Son las teorías las que articulan las categorías de cada elemento sistémico. 


\section{CONCLUSIÓN}

Las dimensiones implicadas con la hipermetropía derivan de la relación naturaleza-cultura, recursos-Estado-contribuyentes, productos-mercadoconsumidores, spots-medios-espectadores y situaciones-participaciones. Se han expuesto dimensiones para explicar las situaciones ambientales a partir de sus relaciones con la cultura, la sociedad, el Estado, la comunidad, el barrio, la familia y el individuo. En este sentido, las situaciones ambientales se derivan conceptualmente de entidades desde los cuales se les puede observar, comparar, analizar y sintetizar. Cuando la humanidad consideró que el agua y ella eran parte de la naturaleza, surgieron los símbolos que la transformaron en culturas. Cuando la humanidad clasificó como recursos al agua, develó al Estado que la transformó en contribuyentes. Cuando la humanidad pensó que el agua era un producto, creó el mercado que la transformó en consumidores. Cuando la humanidad redujo a spots las situaciones ambientales, enalteció a los medios de comunicación que la transformaron en espectadores; y cuando la humanidad comprendió la diversidad de las situaciones ambientales, se autoorganizó para preservar a sus generaciones futuras.

Las relaciones existentes entre la disponibilidad y el consumo mediante procesos sociocognitivos sustentan la hipótesis mediática ambiental en torno a la cual, la cantidad de agua y el uso de la misma están determinadas por ideas preliminares que al ser procesadas en situaciones de abundancia o escasez inhibirán o facilitarán el dispendio o el ahorro de agua sin importar su valor, costo, precio, tarifa, cotización o cualquier otro parámetro que implique restaurar el equilibrio en la disponibilidad y el consumo.

Sin embargo, es necesario reconceptualizar la problemática y establecer parámetros de costo a partir de las necesidades y expectativas humanas independientemente de sus capacidades o bienes. En este sentido, las teorías sociopsicológicas proponen que la medición del consumo de agua se lleve a cabo, ya no en función de su disponibilidad actual o futura, sino en función de las creencias, percepciones, actitudes, conocimientos, valores o intenciones de uso de agua de los individuos insertos en un sistema de abastecimiento. Es decir, las teorías sociopsicológicas solo explican la sustentabilidad de un mercado formal de abastecimiento, pero al referirse a un mercado informal, las teorías encuentran barreras que les impiden explicar la solidaridad en situaciones de escasez o el acaparamiento en situaciones de abundancia.

Por ello, las teorías sociopsicológicas tendrán que explicar las discrepancias que inhiben el desarrollo sustentable de la humanidad en relación a la disponibilidad de agua. En tal sentido, las teorías sociopsicológicas deberán ser complementadas con otras teorías que expliquen la afectividad más que la racionalidad en torno al uso de agua, los grupos a los que el usuario pertenece, los sistemas de cotización en los que el usuario está adscrito o los procesos de gobernanza en los que el ciudadano participe. 


\section{AGRADECIMIENTOS}

Al Consejo Nacional de Ciencia y Tecnología (Conacyt) por el financiamiento de la línea de investigación: "Gobernanza de la Seguridad Pública y Comunicación de Riesgos Sustentables".

\section{POTENCIAL CONFLICTO DE INTERESES}

Los autores declaran no tener ningún conflicto de interés por el orden o contenido del artículo.

\section{FUENTES DE FINANCIACIÓN}

Becas del Consejo Nacional de Ciencia y Tecnología (Conacyt) para estudios de doctorado,

\section{ANEXO}


Tabla 1. Especificación sociopolítica de la hipermetropía

\begin{tabular}{|c|c|}
\hline Dimensión & Definición \\
\hline Psicopatológica global & $\begin{array}{l}\text { "[...] social dilemmas and social representations serve to } \\
\text { move the discussion away from treating global/local } \\
\text { environmental assessment as a form cognitive bias } \\
\text { thought to one of seeing them as a means by which } \\
\text { individuals and social context construct the environment in } \\
\text { order to understand and deal with what is a treating state } \\
\text { of affairs" (Uzzel, 2000, p. 315) }\end{array}$ \\
\hline Ambiental relativa & $\begin{array}{l}\text { "Los problemas ambientales son percibidos como más } \\
\text { graves cuanto más alejados se encuentren del perceptor, } \\
\text { y el sentido de responsabilidad se vuelve impotente ante } \\
\text { problemas percibidos a nivel global. [...] aquellas personas } \\
\text { egocéntricas y/o antropocéntricas reflejarán problemas } \\
\text { ambientales con un impacto local, mientras que las } \\
\text { ecocéntricas o biosféricas tendrán más accesibles } \\
\text { problemas ambientales con un impacto global sobre el } \\
\text { medio ambiente" (Amérigo et al., 2005, p. } 258 \text { ) }\end{array}$ \\
\hline Sustentabilidad divergente & $\begin{array}{l}\text { [...] is a term used to describe to tendency for people to } \\
\text { view broad, distal environmental problems as more severe } \\
\text { than proximate ones. Although it seems logical that } \\
\text { personal connedions and attachment to particular places } \\
\text { would result in enhanced concern at relatively small, local } \\
\text { scales the opposite has been found in several studies" } \\
\text { (Larson et al., 2009, p. 1014) }\end{array}$ \\
\hline Normativa valorativa & 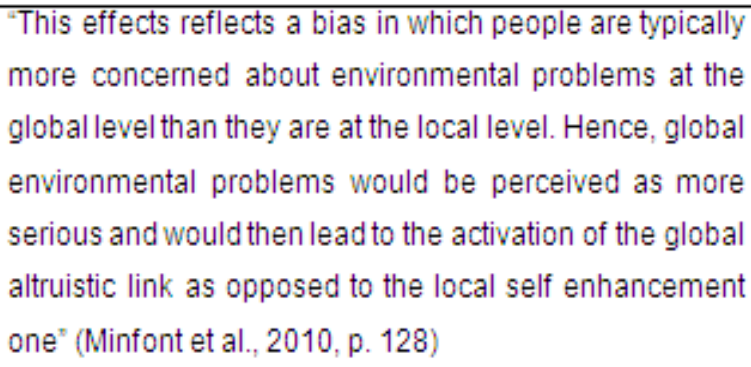 \\
\hline Espacial actitudinal & $\begin{array}{l}\text { [...] cross cultural studies found that people tend to } \\
\text { perceive environmental problems as more serious at the } \\
\text { global than at the local level. [...] research has shown that } \\
\text { individual responsibility for environmental issues is } \\
\text { greatest at the local level and decreased as the spatial } \\
\text { level becomes more distant. Therefore, spatial bias could } \\
\text { hinder pro-environmental behavior because of the lower } \\
\text { sense of responsibility associated with problems at higher } \\
\text { spatial levels" (Tronu, 2011, p. 12) }\end{array}$ \\
\hline
\end{tabular}




\begin{tabular}{|c|c|}
\hline Económica urbana & $\begin{array}{l}\text { "[...] which is defined by the following statement concern } \\
\text { for local environmental issues was more attenuated than } \\
\text { for global ones, risk perception of local sources of pollution } \\
\text { was perceived as lower than distant threats, and global } \\
\text { sources of information about the environment were } \\
\text { considerer more threatening in the global than the local } \\
\text { ones" (Basar, 2011, p. 54). }\end{array}$ \\
\hline Educativa ambiental & $\begin{array}{l}\text { "[...] tendencia a preocuparse por los grandes problemas } \\
\text { ambientales globales, pero no atender }-0 \text { incluso } \\
\text { minimizar-las implicaciones ambientales inmediatas de } \\
\text { las conductas y acciones cotidianas y próximas" (Pol y } \\
\text { Castrechini, 2013, p. 336) }\end{array}$ \\
\hline Política participativa & $\begin{array}{l}\text { "...] public environmental policy can help to alleviate } \\
\text { these problems, but may reduced to irrelevance through } \\
\text { not compliance if not well understood and supported by the } \\
\text { general public. This issue increases with scale, as people } \\
\text { follow the aphorism "think globally, act locally", meaning } \\
\text { that while they tend to be more concerned about global } \\
\text { problems" (Garcia et al., } 2013, \text { p. 136) }\end{array}$ \\
\hline Global ecológica & $\begin{array}{l}\text { "[...] ese fenômeno them correspondência como o } \\
\text { conceito de vies to otimismo ja mencionado, por medio do } \\
\text { qual as pessoas percebem suas localidades de maneira } \\
\text { mais positiva e otimista do que cuando avalian locais } \\
\text { distantes dos seus" (Lucio y Pinheiro, } 2013, \text { p. 180) }\end{array}$ \\
\hline Participativa dialógica & $\begin{array}{l}\text { "[...] the longer term future was seen as more problematic } \\
\text { than the shorter term by participants in these studies, and } \\
\text { global concern were seenas more problematic than local } \\
\text { issues" (Vervoot et al., } 2014, \text { p. } 160 \text { ) }\end{array}$ \\
\hline
\end{tabular}

Fuente: Elaboracion propis.

\section{REFERENCIAS}

- Amérigo, M., Aragonés, J., Sevillano, V. y Cortés, B. (2005). La estructura de las creencias sobre la problemática ambiental. Psicothema, 17(2), 257-262.

- Basar, U. (2011). Environmental psychology, urban planning and economics: intersection crossroad \& tangents. Asian Conference Psychology and the Behavior Sciences. Osaka, Japan.

- Comisión Nacional del Agua. (2008). Estadísticas del Agua en México. México: Conagua. 
- Comisión Nacional del Agua. (2012). Bancos de agua en México. México: Conagua.

- Corral, V., Bonnes, M., Tapia, C., Fraijo, B., Frías, M. y Carrus, G. (2009). Correlates of pro-sustainable orientation the affinity towards diversity. Journal of Environmental Psychology, 29, 3443.

- Corral, V., Fraijo, B. y Pinheiro, J. (2006). Sustainable behavior and time perspective: present, past, and future orientation and their relationships with water conservation behavior. Revista Interamericana de Psicología, 40(2), 139-147.

- Corral, V., Fraijo, B. y Tapia, C. (2008). Un registro observacional del consumo individual de agua; aplicaciones a la investigación de la conducta sustentable. Revista Mexicana de Análisis de la Conducta, 34(1), 79-96.

- Frías, M., Rodríguez, A. y Corral, V. (2009). Análisis de factores que influyen en el desarrollo de normas ambientales y en la conducta anti-ecológica. Revista Interamericana de Psicología, 43(2), 309-322.

- García, C. y Bustos, J. (2013). Los estudios psicológicos de la sustentabilidad hídrica. Aplicaciones al sistema tarifario de consumo. Revista de Ciencias Sociales, 139(1), 65-90.

- García, R., Real, J. y Romay, J. (2005). Temporal and spatial dimensions in the perception of environmental problems: an investigation of the concept of environmental hyperopia. International Journal of Psychology, 40, 5-10.

- García, R., Stea, D., Real, J., Coreno, V. y Elguea, S. (2013). Psychology, participation and environmental policy marking. En García, R. (Coord.), Readings of the Prestige disaster. Contribution from the social sciences (pp. 132-147). Coruña: University of the Coruña.

- Gilford, R. (2009). Temporal pessimism and spatial optimism in environmental assessment. Journal of Environmental Psychology, 29, 1-12.

- Instituto Nacional de Estadística Geografía e Informática. (2010). Mujeres y hombres en México. México: Inegi.

- Larson, K., White, D., Gober, P., Harlan, S. y Wutih, A. (2009). Divergent perspectives on water resource sustainability in a public policy science context. Environmental Science \& Policy, 12, 1012-1023.

- Lucio, H. y Pinheiro, J. (2013). Dimensões ecológicas do aquecimento global conforme a visão adolescentes brasileiros. Estudos de Psicologia, 18(2), 173-182. 
- Milfont, T., Sibley, C. y Duckitt, J. (2010). Testing the moderating role of the components of norm activation on the relationship between values and environmental behavior. Journal of Cross Cultural Psychology, 4(1), 124-131.

- Moser, G. y Uzzell, D. (2004). Psychology and the challenge of global environmental change. International Human Dimensions Programme on Global Environmental Change, 4, 1-4.

- Pol, E. y Castrechini, A. (2013). ¿Disrupción en la educación para la sostenibilidad? Revista Latinoamericana de Psicología, 45(3), 335-349.

- Rathzel, N. y Uzzell, D. (2012). Mending the beach between labour and nature: environmental engagements of trade unions and the North-South divide. Interface, 4(2), 81-100.

- Real, J. y García, R. (2001). Dimensiones de la preocupación ambiental: una aproximación a la hipermetropía ambiental. Estudios de Psicología, 22(1), 87-96.

- Tronu, G. (2011). General environmental attitudes and beliefs: the spatial bias and the NHIP scale. Tesi di Dottorato. Universita di Roma.

- United Nations Habitat. (2010). Sick water? The central role of wastewater management in sustainable. A rapid response assessment. Birkiland: Autor.

- United Nations Water. (2013). Water security \& the global water agenda. Ontario: United Nations University.

- Uzzel, D. (2000). The psychospatial dimension of global environmental problem. Journal of Environmental Psychology, $20,307-318$.

- Uzzel, D. (2004). From local to global. A case of environmental hyperopia. International Human Dimensions Programme on Global Environmental Change, 4, 6-9.

- Uzzell, D., Rice, H., Ballantine, H. y Podlucka, D. (1994). Environmental hyperopia. 13th IAPS Conference, University of Manchester, 13-15 July.

- Vervoort, J., Hoogstra, M., Kok, K., Van Lammeren, R., Bregt, A. y Janssen, R. (2014). Visualizing stakeholder perspectives for reflection and dialogue on scale dynamics in social ecological systems. Human Ecology Review, 20(2), 157-181.

1. Estudios de Doctorado en Psicología Social y Ambiental. Profesor de asignatura, Universidad Autónoma del Estado de México, 
Unidad Académica Profesional Huehuetoca. Huehuetoca, México. garcialirios@uaemex.mx

2. Doctor en Administración. Profesor titular "C", UNAM-ENTS, Distrito Federal, México.javierg@unam.mx

3. Maestro en Educación. Profesor titular "C", UNAM-ENTS, Distrito Federal, México. jorheval@unam.mx

4. Doctor en Psicología. Profesor titular "C", UNAM-FESZ, Distrito Federal, México.marcos.bustos@unam.mx

5. Doctor en Educación. Profesor de tiempo completo, UAEMEXUAPH, Huehuetoca, Estado de México. jaaguilarf@uaemex.mx

Para citar este artículo: García Lirios, C., Carreón Guillén, J., Hernández Valdés, J., Bustos Aguayo, J.M. y Aguilar Fuentes, J.A. (2016). Especificación de un modelo de hipermetropía sociopolítica. Revista Luna Azul, 42 , 270-292.

Recuperado

de http://200.21.104.25/lunazul/index.php?option=com_content\&view=arti cle\&id=139 